\title{
The landscape of transposable elements in the finished genome of the fungal wheat pathogen Mycosphaerella graminicola
}

\author{
Braham Dhillon ${ }^{1}$, Navdeep Gill'², Richard C Hamelin ${ }^{1,3}$ and Stephen B Goodwin ${ }^{4^{*}}$
}

\begin{abstract}
Background: In addition to gene identification and annotation, repetitive sequence analysis has become an integral part of genome sequencing projects. Identification of repeats is important not only because it improves gene prediction, but also because of the role that repetitive sequences play in determining the structure and evolution of genes and genomes. Several methods using different repeat-finding strategies are available for whole-genome repeat sequence analysis. Four independent approaches were used to identify and characterize the repetitive fraction of the Mycosphaerella graminicola (synonym Zymoseptoria tritici) genome. This ascomycete fungus is a wheat pathogen and its finished genome comprises 21 chromosomes, eight of which can be lost with no obvious effects on fitness so are dispensable.

Results: Using a combination of four repeat-finding methods, at least $17 \%$ of the M. graminicola genome was estimated to be repetitive. Class I transposable elements, that amplify via an RNA intermediate, account for about $70 \%$ of the total repetitive content in the M. graminicola genome. The dispensable chromosomes had a higher percentage of repetitive elements as compared to the core chromosomes. Distribution of repeats across the chromosomes also varied, with at least six chromosomes showing a non-random distribution of repetitive elements. Repeat families showed transition mutations and a CpA $\rightarrow$ TpA dinucleotide bias, indicating the presence of a repeat-induced point mutation (RIP)-like mechanism in M. graminicola. One gene family and two repeat families specific to subtelomeres also were identified in the M. graminicola genome. A total of 78 putative clusters of nested elements was found in the M. graminicola genome. Several genes with putative roles in pathogenicity were found associated with these nested repeat clusters. This analysis of the transposable element content in the finished M. graminicola genome resulted in a thorough and highly curated database of repetitive sequences.

Conclusions: This comprehensive analysis will serve as a scaffold to address additional biological questions regarding the origin and fate of transposable elements in fungi. Future analyses of the distribution of repetitive sequences in M. graminicola also will be able to provide insights into the association of repeats with genes and their potential role in gene and genome evolution.
\end{abstract}

\section{Background}

Mycosphaerella graminicola (synonym Zymoseptoria tritici, the causal agent of septoria tritici blotch, STB) poses a worldwide threat to wheat production, with yield losses of up to $30-40 \%$ or more during years with severe epidemics [1]. Although the use of fungicides and deployment of resistant wheat cultivars can help to contain

\footnotetext{
* Correspondence: sgoodwin@purdue.edu

${ }^{4}$ USDA-ARS, Crop Production and Pest Control Research Unit, Purdue University, 915 W. State Street, West Lafayette, Indiana 47907-2054, USA Full list of author information is available at the end of the article
}

M. graminicola losses in the field, breeding for resistance to STB has been slow and the resistance often is not durable [2]. With the rapid evolution of fungicide resistance in M. graminicola populations [3,4] and failure of resistance genes in the field [2], there is an urgent need for improved measures to control STB.

Toward this end, availability of the M. graminicola genome, sequenced to completion by the Department of Energy - Joint Genome Institute (DOE-JGI) [5], is a valuable resource that may be utilized for developing better disease-control strategies. This can be achieved by

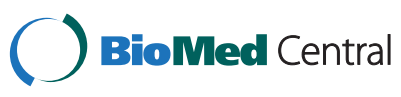


identifying and characterizing the genomic components that may have an effect on the disease-causing abilities of the pathogen. Besides specific genes involved in pathogenicity and host specificity, intergenic regions and repetitive sequences, especially transposable elements (TEs), also influence the structure, function and regulation of genes.

Repetitive sequences are those that exist more than once in a genome, and are now known to be common features of eukaryotic genomes. Repetitive sequences include gene families, pseudogenes, segmental duplications, tandem repeats and transposable elements. Transposable elements, also known as mobile elements, are a special class of repetitive sequences that can move from one locus to another in a genome, either encoded proteins required for their own movement (autonomous TEs) or dependent on other autonomous elements for their movement (nonautonomous TEs). During the process of TE integration at a new genomic site, a few nucleotides flanking the new insertion site are duplicated creating a target site duplication (TSD), which is a signature for TE insertion/excision [6].

TEs can be divided into two main categories based on their mode of replication: Class I TEs or Retrotransposons; and Class II TEs or DNA transposons that also include the Miniature Inverted-repeat Transposable Elements (MITEs). Retrotransposons typically include coding sequences for several proteins including a reverse transcriptase that transcribes the RNA to a cDNA, which is integrated back into the genome, thereby following a copy-paste mechanism to move to a new genomic location. Retrotransposons can be further classified as Long Terminal Repeat (LTR) retrotransposons, which carry long terminal repeats at both ends, and Non-LTR retrotransposons, that lack LTRs but have a poly-A tail at their 3' end. Class II (DNAbased) transposons, on the other hand, follow a cut-paste mechanism and move to a new genomic location without an RNA intermediate. DNA transposons typically are delimited by terminal inverted repeats (TIRs) and encode a transposase domain. Transposon-encoded transposase recognizes the TIRs, excises the element and integrates it into a new location [6]. Helitrons and cryptons are also classified as DNA transposons although they lack the traditional TIRs. Occurrence of distinct structural features and protein domains can be used to identify and distinguish between the different classes of TEs in a genome [6].

Since their discovery during the late 1940s by Barbara McClintock [7], the perceived importance of repetitive sequences has undergone a fundamental change from being considered inert components of the genome to drivers of genome evolution [8]. Identification of TEs is important to understand their functional significance, as they have the ability to alter the function and structure of the genome. For example, comparative genome analysis revealed that the three-fold genome size expansion of the oomycete Phytophthora infestans as compared to P. ramorum was mediated by TEs [9], altering the genome structure by creating gene-rich islands separated by vast expanses of repetitive sequences. Besides affecting the genome structure, TEs have the ability to create new genes $[8,10]$ and to modulate the function of existing genes to create new phenotypes [11]. An example of the latter phenomenon has already been documented in the $M$. graminicola genome, where a single-copy DNA methyltransferase gene was duplicated into a subtelomeric region and then amplified among the telomeres to a dozen copies, all of which were subsequently recognized by the repeat-induced point mutation (RIP) machinery and inactivated, including the original copy [12]. This led to a loss of cytosine methylation in $M$. graminicola, although the RIP machinery appears to be intact [12].

A survey of the completely sequenced $M$. graminicola genome was done to identify and categorize repetitive sequences, especially TEs, and to determine their chromosomal locations, both as independent and nested insertions. These data, when combined with the genome annotation [5], will help determine the association of TEs with the genic regions and further our understanding of TE-mediated processes that may be involved in the regulation of gene function.

\section{Results}

\section{Identification of repetitive sequences}

The 39.7-Mb genome of $M$. graminicola was sequenced to completion by the DOE-JGI; telomere-to-telomere sequence information is available for all but chromosome 18 , which is missing two gaps of unclonable DNA (sizes of 1.4 and $4.5 \mathrm{~kb}$ ), and chromosome 21 , which is missing one telomere [13]. With only three gaps, it is the most finished genome for a filamentous fungus and comprises 21 chromosomes that carry 10,952 predicted genes [13]. The chromosomes have been arranged and named in order of their decreasing lengths. The M. graminicola genome is highly plastic as it can randomly lose up to eight of the smallest chromosomes (numbers 14-21), aptly labeled as dispensable [14].

Four strategies were employed to estimate the repetitive content of the $M$. graminicola genome. Initially, only $1.1 \%$ of the $M$. graminicola genome was identified as being repetitive when using RepeatMasker (RM) [15] with the default RepBase Update [16] repeat library containing fungal-specific repeats. This led us to identify repeats $a b$ initio to compile and annotate a custom repeat library, for which RECON [17] was used. When this custom repeat library was used with RM [15], discovery of divergent elements increased the estimated repetitive content of the $M$. graminicola genome to $16.7 \%$ (6.7 Mb) (Table 1). This repetitive fraction does not reflect the tandem repeats (low-complexity and simple sequence) and 
Table 1 Predicted repetitive content in the Mycosphaerella graminicola genome

\begin{tabular}{lcc}
\hline \multicolumn{1}{c}{ Method } & $\begin{array}{c}\text { Repetitive } \\
\text { bases }\end{array}$ & $\begin{array}{c}\text { Percent } \\
\text { repetitive }\end{array}$ \\
\hline RECON, parsed & $4,629,313$ & 11.7 \\
RepeatMasker - RepBase Update & 467,778 & 1.2 \\
RepeatMasker - RECON, parsed & $6,921,597$ & 17.4 \\
RepeatMasker - RECON, parsed, nolow* & $6,634,996$ & 16.7 \\
RepeatMasker - RepeatScout & $7,400,585$ & 18.6 \\
\hline
\end{tabular}

*RepeatMasker nolow option, does not mask the low-complexity DNA or simple repeats.

low-copy repetitive families with fewer than ten repeats per family; there were 2,500 low-copy repeat families in total that accounted for 2,279,215 bp (5.7\%) of the $M$. graminicola genome. RepeatScout [18], another tool for de novo repeat identification, was used to create a second custom repeat library which, in conjunction with RM [15], identified $18.7 \%(7.4 \mathrm{Mb})$ of the M. graminicola genome as repetitive (Table 1). A k-mer based approach, TALLYMER [4], was also used to identify and plot repeats across all of the chromosomes. The repeats predicted by different methods have been shown as separate tracks (Figure 1) for an essential (chromosome 8) and a dispensable chromosome (chromosome 14). The core chromosomes contain $88 \%$ of the genome and $79 \%$ of the repetitive fraction, whereas, the remaining $21 \%$ of the repeats are present on the $12 \%$ of the genome contained in the dispensable chromosomes. In general, the dispensable set of chromosomes had a statistically significantly higher $(t=6.1292$, $\mathrm{P}<0.0001)$ repetitive content compared to the corechromosome set (Figure 2).

\section{Repeat annotation}

The repeat families identified by RECON [17] were categorized into six major classes (Table 2). Based on the repeat copy number, 105 families of high-copy repeats (10 or more copies in the genome) were annotated in detail in the M. graminicola genome. However, based on their distribution in the genome and sequence overlap, 12 families were merged with other families, decreasing the effective number of families to 93 with copy numbers ranging from 7 to 272 (Additional file 1: Table S1). Among the 2,500 low-copy repetitive families, only 125 families occupying 525,399 bp (1.3\%) of the M. graminicola genome could be annotated (Additional file 2: Table S2). Among the different repeat classes, retrotransposons were the most common in the M. graminicola genome. In total, 21 LTR retrotransposon and four non-LTR retrotransposon families were identified. Class I TEs (both LTR and non-LTR retrotransposons) in total comprised $70.5 \%$ of the repetitive fraction in the genome. LTRs and TSDs could only be determined for 15 LTR retrotransposon families; the remaining 6 families had other characteristics of LTR retrotransposons except for the LTR. LTR lengths ranged from 110 to $378 \mathrm{bp}$ and length of the TSDs varied from 4-5 bp. Average insertion age for the LTR retrotransposons was estimated to be $2.4 \pm 1$ Million years (My), with the oldest insertion event clocked at 5.6 My (Figure 3).

Unclassified repeats, those with structural features characteristic of the major groups but with no similarity to protein domains or structural features associated with sub-groupings of known repeats, was the next major category. Thirty-seven families of unclassified repeats were identified that occupied $14.9 \%$ of the repetitive fraction.

Fifteen families of non-MITE DNA transposons, thirteen MITE families and three families of helitrons (helicase domain-containing repeats) were also identified in the genome. Helitrons are atypical DNA transposons as they lack TIRs and do not generate a TSD upon integration. Three helitron-associated structural features, i.e., dinucleotide TA at the $5^{\prime}$ end, hair-pin loop followed by the tetranucleotide 'CTRR' at the 3' end, were present in two families (families 98 and 765); only the hair-pin loop could be identified in the third family (family 637). DNA transposons in total accounted for $14.6 \%$ of the repetitive DNA. Among the thirteen MITE families discovered, nine had 2-bp TSDs (TA: 8 families; CT: 1 family), two had 5 -6-bp TSDs and one family had an 8 -9-bp TSD. Based on having the same TIR and TSD but different internal sequences, four (families 55, 112, 222 and 290) and three MITE families (246, 298 and 2546) could be grouped into two superfamilies.

\section{Distribution of repeats across the chromosomes}

A non-parametric runs test was used to check the randomness of repetitive sequence distribution across the M. graminicola chromosomes. Chromosomes were initially partitioned into bins of $100 \mathrm{~kb}$ each. Each bin was scored as 1 or 0 , if the repetitive content of the bin was higher or lower, respectively, compared to the mean repeat content of the chromosome. A survey of these 100$\mathrm{kb}$ bins showed that repetitive sequences on two core chromosomes, 8 and 10, had a nonrandom, clustered distribution. Because the number of runs was below the threshold value of 10 for all of the dispensable chromosomes and one core chromosome (number 13), we repeated the survey for smaller bins (50 kb). Using 50-kb bins, four additional chromosomes (core chromosome 6 and dispensable chromosomes 17, 18 and 19) showed a non-random distribution of repetitive sequences (Table 3; Additional file 3: Table S3). The distribution of genes also was tested in the smaller bins and showed a nonrandom pattern on chromosomes 8 (core chromosome) and 15 (dispensable chromosome). 


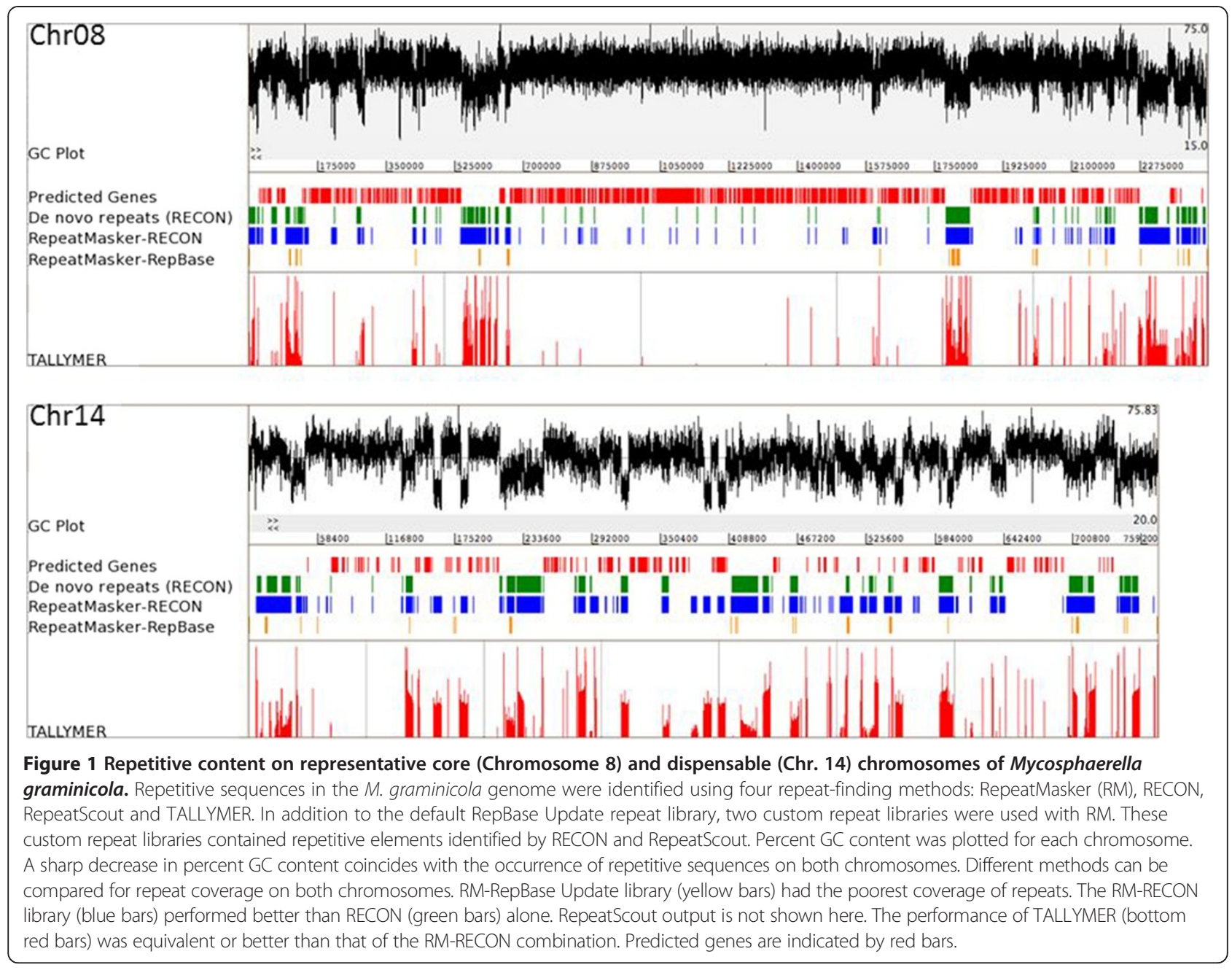

Randomness of inter-element distances also was tested. Using the Shapiro-Wilk test, the null hypothesis that repeats are distributed randomly was rejected and the $\mathrm{P}$ values were highly significant for all $M$. graminicola chromosomes (results not shown). Therefore, this analysis does not seem to discriminate as well as the nonparametric means test.

\section{Nested blocks of elements}

Repetitive elements inserted into other elements are described as nested. A nested cluster is initiated when a TE inserts (primary insertion event) into the base element (element in which the primary insertion occurs). There can be multiple primary insertions into the base element, as a new independent insertion event can occur adjacent to the existing insertion. A total of 78 putative nested element clusters was identified in the M. graminicola genome, with 69 (88\%) clusters present on the core chromosomes. Their length ranged from 7.5 to $63 \mathrm{~kb}$, with 33 clusters being greater than $20 \mathrm{~kb}$. These clusters account for about a quarter $(24 \%)$ of the total repetitive content in the genome. The number of different elements in a cluster varied from 2 to 7 . An LTR retrotransposon was the base element in 49 clusters (Figure 4), out of which TSDs were verified in 35 clusters (Table 4). In eight clusters (length greater than $20 \mathrm{~kb}$ ) with a DNA transposon or an unclassified element as the base element, TSDs were verified for the primary LTR retrotransposon insertion event. In general, the primary insertion event was dominated by LTR retrotransposons, with 59 such cases identified. One non-LTR retrotransposon family (family 623), found in 30 primary insertion events, was noticeably absent from all nested structures with a DNA transposon as the base element.

Genes also were found associated with the clusters of transposable elements. Sixteen genes (including kinases, peptidases and cytochrome c) were found inserted in the clusters and 60 genes were found in the close vicinity of these nested clusters (within $2 \mathrm{~kb}$ of the cluster boundary). Eleven of these genes contained signal peptides 


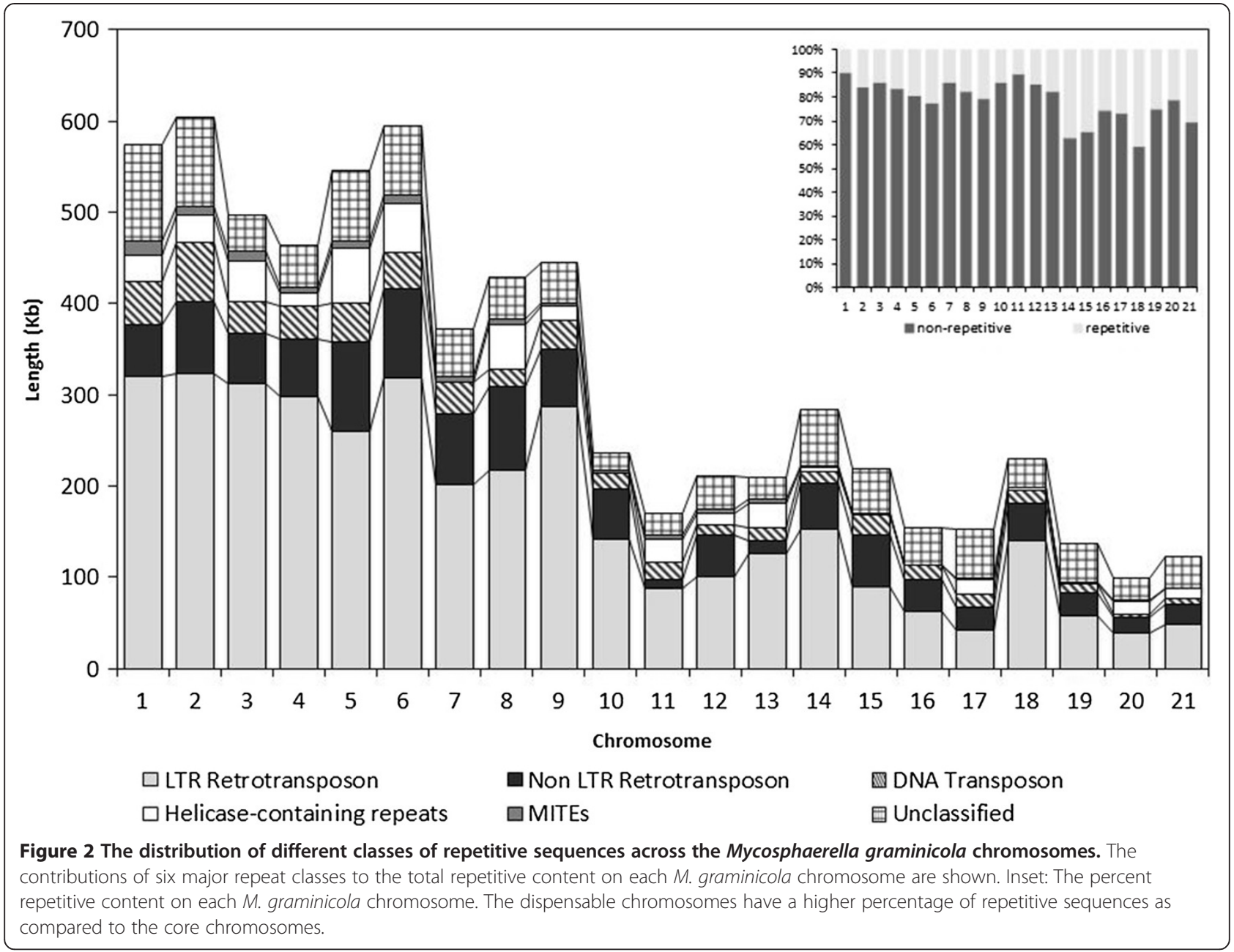

and were potentially secreted, and others included genes encoding enzymes such as hydrolases, kinases, cytochromes, and chloroperoxidases.

\section{Subtelomeric regions}

The length of a subtelomere was defined as a continuous stretch of repetitive DNA starting from the telomeric repeats, without any intervening unique DNA. Different chromosomes had varying lengths of subtelomeric repeats (Figure 5). The length of subtelomeric regions varied from $1.6 \%$ (chromosome 20) to $91.4 \%$ (chromosome 1) of the $100-\mathrm{kb}$ sequence that was analyzed from the end of each chromosome. Although the repeats in subtelomeric regions did not show a biased distribution, some chromosomes had subtelomeric regions that were highly similar to sub-telomeres on other chromosomes. Long stretches of highly similar subtelomeric sequences were shared between three pairs of core and dispensable chromosomes, chromosomes 2 and $17(58 \mathrm{~kb}), 9$ and $21(19 \mathrm{~kb})$ and 4 and $18(7 \mathrm{~kb})$, and one pair of core chromosomes, 8 and $13(37 \mathrm{~kb})$.
Two repeat families were limited to subtelomeric regions only. One was a family of non-LTR retrotransposons and the other was a repeat family containing a DNA methyltransferase domain [12], atypical of repetitive elements. The non-LTR retrotransposon family (family 4) that was limited to sub-telomeric regions contained 34 full-length elements and 49 truncated elements. Depending on the number of tandem repeat copies in the non-LTR element, the full-length elements were $3.4-3.5 \mathrm{~kb}$ long. These full-length elements were present on 16 out of the 21 chromosomes and on 26 of the 41 chromosome ends. Thirteen cases were identified on different chromosomes where a truncated element was found in tandem to the full-length element. One to three copies of the telomeric hexamer repeat TTAGGG were present at the $3^{\prime}$ end of all the full-length and 38 truncated elements. The telomeric repeat-containing 3' end of the non-LTR element was always pointing away from the telomere. Therefore, the orientation of the elements on one end of the chromosome was the same whereas elements on the other chromosome end were 
Table 2 Classification of Mycosphaerella graminicola repetitive families identified de novo by using RECON

\begin{tabular}{|c|c|c|c|c|c|}
\hline Class & Repeat class (\# families) & Repeat type & Total length (bp)* & Percent of repetitive content & Percent of genome \\
\hline \multirow[t]{3}{*}{ Class I } & LTR Retrotransposon (21) & Ty1-Copia & $1,039,062$ & 15.7 & 2.6 \\
\hline & & Ty3-Gypsy & $1,803,457$ & 27.2 & 4.5 \\
\hline & & Unclassified & 766,193 & 11.5 & 1.9 \\
\hline \multirow[t]{3}{*}{ Subtotal } & & & $3,608,712$ & 54.4 & 9.1 \\
\hline & Non LTR Retrotransposon (4) & Tad1-like & 697,083 & 10.5 & 1.8 \\
\hline & & Unclassified & 371,533 & 5.6 & 0.9 \\
\hline Subtotal & & & $1,068,616$ & 16.1 & 2.7 \\
\hline \multirow[t]{7}{*}{ Class II } & DNA Transposon (15) & CACTA En/Spm & 78,909 & 1.2 & 0.2 \\
\hline & & hAT & 70,346 & 1.1 & 0.2 \\
\hline & & Tc1-Mariner & 58,977 & 0.9 & 0.1 \\
\hline & & Tc5/Pogo & 44,941 & 0.7 & 0.1 \\
\hline & & Unclassified & 228,727 & 3.4 & 0.6 \\
\hline & MITEs (13) & & 98,726 & 1.5 & 0.2 \\
\hline & Helitron (3) & & 390,961 & 5.9 & 1.0 \\
\hline Subtotal & & & 971,587 & 14.6 & 2.4 \\
\hline Unclassified & Unclassified (37) & Unclassified & 986,081 & 14.9 & 2.5 \\
\hline Total & & & $6,634,996$ & & 16.7 \\
\hline
\end{tabular}

*These repeat estimates were calculated using families containing at least 10 members.

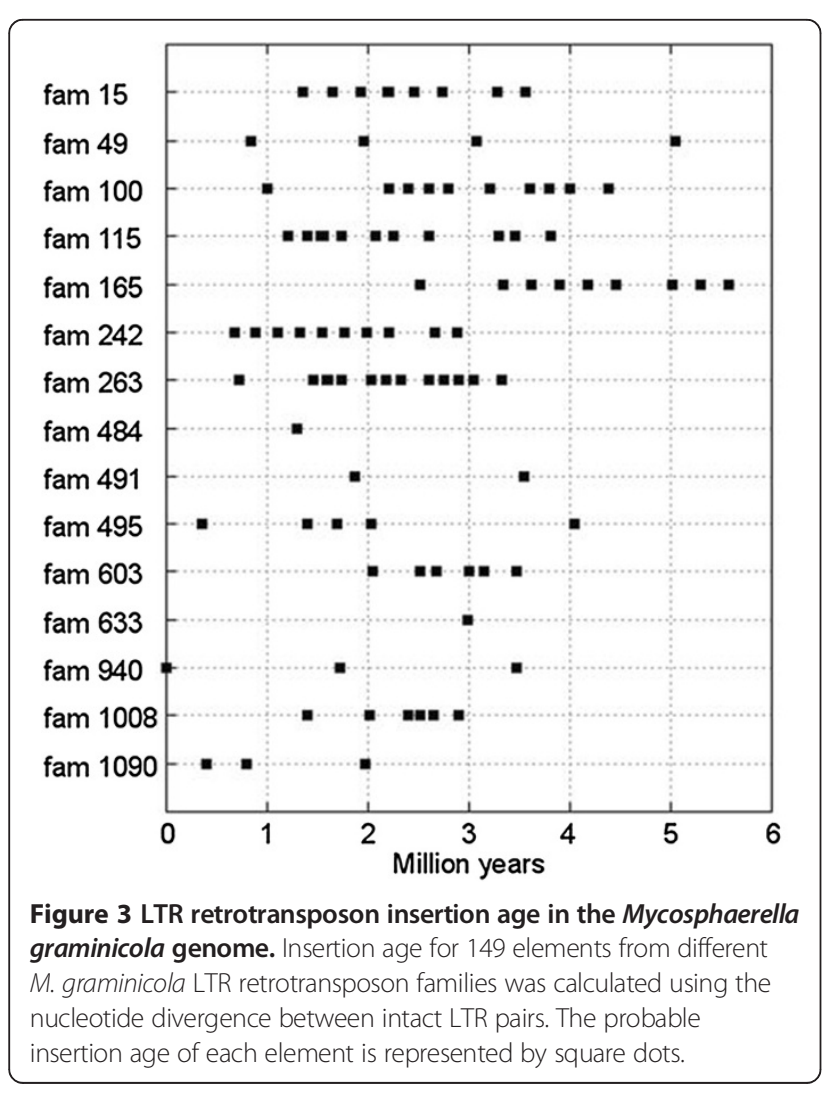

present in the opposite orientation. Telomere-associated repetitive elements with similar characteristics, i.e., containing a reverse-transcriptase gene and terminating in telomeric repeats at their 3' ends, have been described previously as Penelope-like elements (PLEs) [19].

Besides telomere-associated repeats, one class of telomere-associated gene also was found (Figure 5). Initially, a large open reading frame (ORF) of $\sim 3.2 \mathrm{~kb}$ was identified in the subtelomeric region of chromosome 12. Upon further annotation, the putative coding sequence was 3,480 bp long with a very low GC content of $34.7 \%$. Similarity searches identified 13 copies (7 full length, 6 truncated) on nine chromosomes in the genome. A search of the NCBI non-redundant database revealed similarity to subtelomeric RecQ helicase from Schizosaccharomyces japonicus (at 5e-11). One pair of RecQ helicases was a part of the above-mentioned stretch of similar subtelomeric sequences between core chromosomes 8 and 13 .

\section{Tandem repeats}

Based on their repeat unit lengths, tandem repeats are broadly classified into three categories: microsatellites (1-6 nucleotides); minisatellites (7-100 nucleotides); and satellites (>100 nucleotides). All dispensable chromosomes had a higher percentage of tandem repeats. An increase in minisatellite content contributed to this difference between core and dispensable chromosomes (Figure 6). Chromosomes 9 and 13 had an unusually higher percentage of satellite repeats as compared to other core chromosomes. 
Table 3 Randomness of repetitive sequence distribution along each Mycosphaerella graminicola chromosome using a non-parametric runs test

\begin{tabular}{|c|c|c|c|c|c|c|c|c|c|c|c|c|c|c|c|c|c|c|c|c|c|}
\hline \multirow[b]{2}{*}{ Statistic } & \multicolumn{21}{|c|}{ Chromosome } \\
\hline & 1 & 2 & 3 & 4 & 5 & 6 & 7 & 8 & 9 & 10 & 11 & 12 & 13 & 14 & 15 & 16 & 17 & 18 & 19 & 20 & 21 \\
\hline$n$ & 122 & 78 & 70 & 58 & 58 & 54 & 54 & 49 & 43 & 34 & 33 & 30 & 24 & 16 & 13 & 13 & 12 & 12 & 11 & 10 & 9 \\
\hline $\mathrm{n} 1$ & 35 & 26 & 23 & 17 & 24 & 22 & 18 & 18 & 17 & 12 & 10 & 13 & 9 & 6 & 6 & 5 & 4 & 4 & 6 & 6 & 4 \\
\hline no & 87 & 52 & 47 & 41 & 34 & 32 & 36 & 31 & 26 & 22 & 23 & 17 & 15 & 10 & 7 & 8 & 8 & 8 & 5 & 4 & 5 \\
\hline Runs & 55 & 36 & 31 & 21 & 29 & $22^{+}$ & 19 & $15^{\dagger}$ & 19 & $11^{\dagger}$ & 13 & 13 & 7 & 10 & 7 & 6 & $5^{+}$ & $7^{\dagger}$ & $5^{\dagger}$ & 4 & 6 \\
\hline$P$ value & & & & & & $2.7 e^{-2}$ & & $1.9 e^{-6}$ & & $1.3 e^{-4}$ & & & & & & & $1.5 e^{-2}$ & $1.5 e^{-2}$ & $2.7 e^{-2}$ & & \\
\hline
\end{tabular}

Each chromosome was divided into non-overlapping 50-kb bins. Repetitive content in each bin was compared to the chromosomal average repetitive content and each bin was scored either 1 (bin repetitive content greater than chromosomal average) or 0 (bin repetitive content lower than chromosomal average). Each consecutive occurence of $0 \mathrm{~s}$ and $1 \mathrm{~s}$ was calculated as a run. $\mathrm{n}$, the total number of observations; $\mathrm{n} 1$, count of occurrences of ones; n0, count of occurrences of zeros; Runs, number of consecutive occurrences or runs of ones and zeros. ${ }^{\dagger} \mathrm{P}<0.0$.

As tandem repeats often occur in genes, tandem repeat content of both unmasked and masked M. graminicola genome was compared. In the unmasked sequence, 225 tandem repeat families (7,509 repeats) covering $556 \mathrm{~kb}$ of the genome were found. The repeat unit size varied from 1 to $1950 \mathrm{bp}$. On the other hand, the number of tandem repeat families in masked sequence was reduced to 195 (5,109 repeats) and the repeat unit size varied from 1 to $479 \mathrm{bp}$. Tandem repeats in the masked genome covered a total of $374 \mathrm{~kb}$ with $107 \mathrm{~kb}(29 \%)$ being a part of the M. graminicola gene space. Thus,

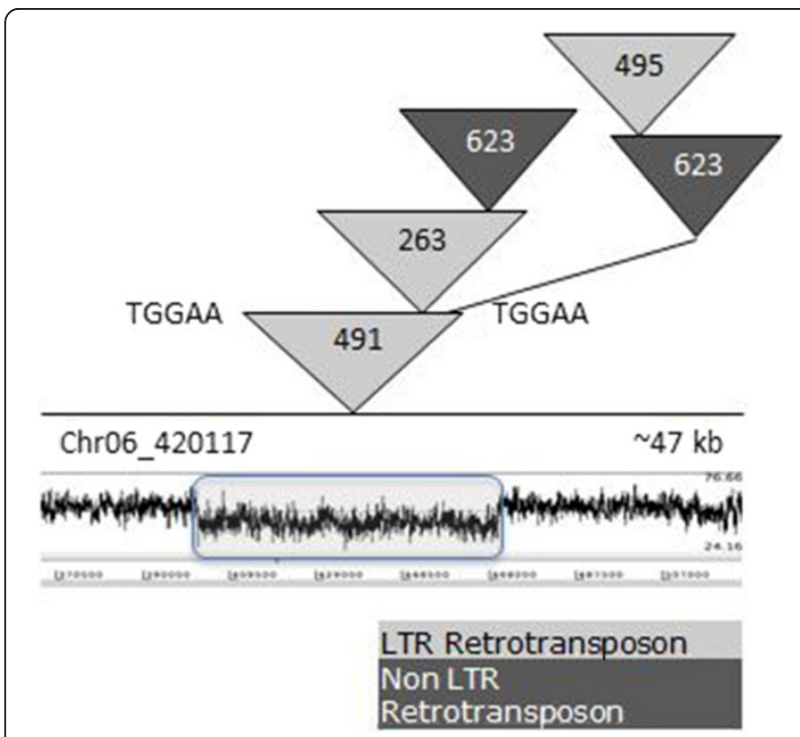

Figure 4 A nested cluster of transposable elements on Mycosphaerella graminicola chromosome 6. A 47-kb nested cluster with an LTR retroelement as the base is indicated. It has a 5-bp target-site duplication (TGGAA). Each triangle represents one insertion and the numbers inside the triangle refer to the repeat family. This cluster only had class I elements. These elements have been shaded as follows: light grey, LTR retrotransposons; dark grey, Non-LTR retrotransposons. The bottom panel shows the percent GC plot for the corresponding repeat region. A decrease in percent GC content in the repeat region is evident when compared to the flanking DNA sequence. about one-third of the tandem repeats identified in the masked genome were components of genes, as the repetitive fraction was masked out prior to analysis.

\section{Extent of repeat-induced point mutations (RIP)}

All repeat families showed elevated levels of transition mutations indicating RIP (Additional file 1: Table S1). A clear $\mathrm{CpA} \rightarrow \mathrm{TpA}$ dinucleotide bias was detected in 70 repeat families (Figure 7), while the remaining 24 families (10 MITEs and 14 unclassified repeat families) failed to show a specific dinucleotide bias. Although the analysis only looks at one strand, both $\mathrm{C} \rightarrow \mathrm{T}$ and $\mathrm{G} \rightarrow \mathrm{A}$ polymorphisms on that strand were quantified to account for RIP on the complementary strand. All of the unclassified repeats and these ten MITE families were less than $200 \mathrm{bp}$ in their average match length. A pairwise comparison of repeats using the highest-GC element in each family as a reference sequence showed that the identity between elements in the same family ranged from 83 to $99 \%$. However, two 6.7-kb sequences were identified on chromosome 7 that were $100 \%$ identical. These sequences contain the rDNA repeats, only two copies of which could be assembled in the released genome sequence.

\section{Active elements}

Almost all elements analyzed carried numerous mutations suggesting the presence of a RIP-like mechanism in M. graminicola. However, elements belonging to at least one family of non-LTR retrotransposons (family 623) and one family of copia-type LTR retrotransposons (family 18) were found in the genome that carried minimal RIP-signature mutations.

Using the reverse transcriptase domain from the nonLTR retrotransposon, at least 119 similar sequences were detected in the genome. All of these sequences, except 11 , carried one or more stop codons. The remaining 11 sequences also had many transition mutations, but none of them resulted in a stop codon in the reversetranscriptase domain. These 11 elements had 90-95\% 
Table 4 Nested clusters of repetitive elements in the Mycosphaerella graminicola genome with an LTR retrotransposon as base element and a verified target site duplication (TSD)

\begin{tabular}{|c|c|c|c|c|c|c|}
\hline Chr & Cluster start & Cluster end & Base family & Cluster size (bp) & Target site duplication & Internal family ${ }^{\S}$ \\
\hline 1 & 910,151 & 924,049 & 49 & 13,898 & GGTAG & 15 \\
\hline 1 & $3,757,104$ & $3,770,311$ & 115 & 13,207 & CCTAC & 263 \\
\hline 2 & 162,839 & 175,222 & 242 & 12,383 & CTTAG & 49 \\
\hline 2 & 811,149 & 825,327 & 242 & 14,178 & CTGTG & 623 \\
\hline 2 & $1,675,912$ & $1,693,177$ & 15 & 17,265 & CGAC & 242 \\
\hline 2 & $1,868,450$ & $1,894,757$ & 263 & 26,307 & AAGGT/C & $(2477(495,623))$ \\
\hline 2 & $3,605,891$ & $3,620,926$ & 15 & 15,035 & ACAGG & 623 \\
\hline 3 & 575,530 & 603,863 & 940 & 28,333 & ATTGA & $(263(623,115))$ \\
\hline 3 & 787,112 & 832,923 & 495 & 45,811 & CTAC & $(2477,623,633(623), 49(115))$ \\
\hline 3 & $1,573,516$ & $1,594,725$ & 495 & 21,209 & CCAG & $(1045,2477)$ \\
\hline 3 & $2,416,365$ & $2,433,023$ & 15 & 16,658 & CTGC & 623 \\
\hline 4 & $1,534,719$ & $1,552,726$ & 100 & 18,007 & ATTAG & 609 \\
\hline 4 & $1,567,363$ & $1,594,101$ & 491 & 26,738 & ATAC & $(603,623)$ \\
\hline 5 & 192,764 & 204,730 & 165 & 11,966 & TाTG & 623 \\
\hline 6 & 191,008 & 210,165 & 242 & 19,157 & ATGA & $(623,242)$ \\
\hline 6 & 420,117 & 467,482 & 491 & 47,365 & TGGAA & $(263(623), 623(495))$ \\
\hline 6 & 771,418 & 802,180 & 491 & 30,762 & GGATG/GGCCG & $623(\times 3)$ \\
\hline 6 & $2,542,200$ & $2,570,636$ & 100 & 28,436 & CATTG & $(623(495))$ \\
\hline 7 & $1,261,818$ & $1,284,705$ & 633 & 22,887 & GAGCT & 495 \\
\hline 7 & $1,787,729$ & $1,808,698$ & 165 & 20,969 & GCATC & 495 \\
\hline 7 & $1,821,242$ & $1,835,308$ & 603 & 14,066 & GCATA/G & 623 \\
\hline 7 & $2,535,616$ & $2,549,001$ & 242 & 13,385 & ATATC & 623 \\
\hline 9 & 64,037 & 77,995 & 49 & 13,958 & GATAG & 623 \\
\hline 9 & 79,932 & 109,217 & 603 & 29,285 & CATTGG & 623 \\
\hline 9 & 962,581 & 977,124 & 49 & 14,543 & CCAAT & 1008 \\
\hline 9 & $1,052,214$ & $1,078,572$ & 242 & 26,358 & TATGA & $(49(263(623)))$ \\
\hline 10 & 863,891 & 879,094 & 633 & 15,203 & GCTGC & 242 \\
\hline 10 & $1,306,971$ & $1,330,127$ & 165 & 23,156 & AGATA & $(623(623), 623)$ \\
\hline 12 & 609,353 & 623,337 & 940 & 13,984 & GCTTC & 49 \\
\hline 13 & $1,028,648$ & $1,051,790$ & 100 & 23,142 & G/CAAAG & 495 \\
\hline 14 & 411,612 & 433,895 & 603 & 22,283 & TTAAG & 495 \\
\hline 14 & 587,485 & 599,441 & 165 & 11,956 & CGGTT & 623 \\
\hline 15 & 192,089 & 204,601 & 242 & 12,512 & GCTIT & 623 \\
\hline 16 & 510,094 & 526,146 & 15 & 16,052 & TTCT & 263 \\
\hline 18 & 294,431 & 312,546 & 242 & 18,115 & GAAGA & $(623,623)$ \\
\hline
\end{tabular}

Columns: Chr, Chromosome. ${ }^{\S}$ The brackets represent the level on nesting.

sequence identity with each other and their RT domains were 97.5 - 99.9\% identical. Also, a 12-bp TSD was identified for eight of the 11 sequences. This non-LTR retrotransposon is specific to fungi and belongs to the Tad-1 clade of non-LTR retrotransposons. A total of 22 repeatderived EST sequences had 100\% identity to 21 elements from this non-LTR family. Another LTR retrotransposon (family 242) was also represented in the EST dataset.
The LTR retrotransposon family with minimal evidence of RIP had only one complete copy that was present on chromosome 18. The element was 5,545 bp long with 515-bp LTRs and a 4-bp TSD (ACTT) (Additional file 2: Table S2). LTR alignment showed two mismatches, both transition mutations, which indicates a recent transposition event, as LTR sequences keep accumulating mutations with age. There was one truncated copy on 


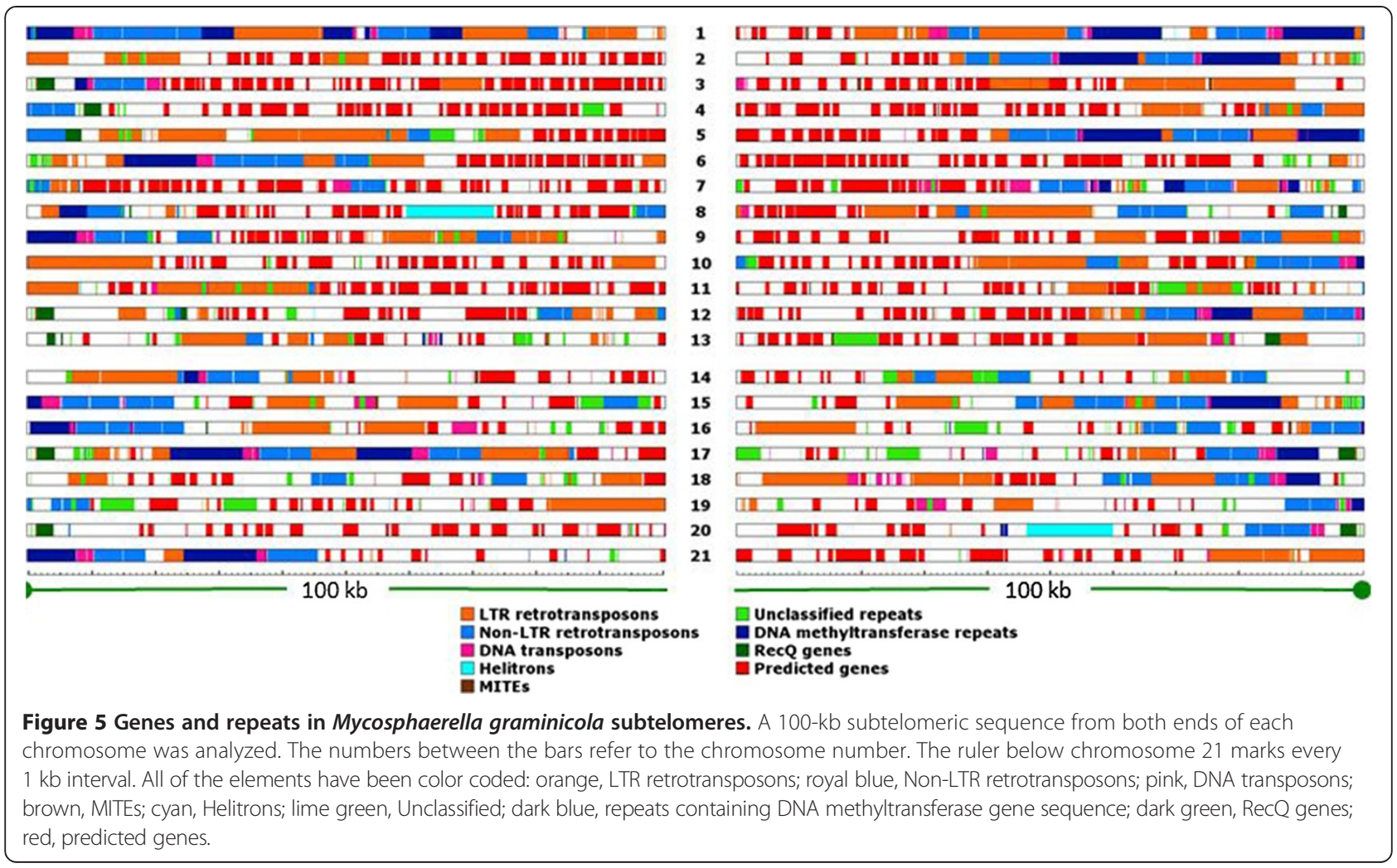

chromosome 17 and two soloLTRs, one each on chromosomes 8 and 12 .

\section{Discussion}

\section{Repeat identification strategies}

With the availability of faster and cheaper sequencing technologies, the real challenge is not sequencing a genome but its annotation. Early identification and analysis of repetitive sequences sets the ground for a better annotation of the genic and inter-genic regions. Several approaches were used for repetitive sequence analysis in the completed M. graminicola genome. Search strategies that exploit structural features to identify and classify TEs in sequenced genomes are known as similaritybased methods. RepeatMasker [15], a similarity-based approach, identifies repetitive elements based on their similarity to already known repetitive sequences. Besides similarity-dependent methods, two other approaches, de novo and k-mer based, can be used to define repetitive elements in a genome. Methods such as RECON [17] utilize whole-genome alignments to identify repetitive elements de novo. K-mer-based methods such as RepeatScout [18] and TALLYMER [20] calculate the frequency of oligomers of different lengths to delineate repetitive sequences in the genome. Since the k-mer and de novo methods do not rely on the existing repeat datasets, they are also suitable for identifying novel repeats in a genome.
With the default RepBase Update [16] repeat dataset comprising all of the fungal-specific repetitive elements only $\sim 1 \%$ of the $M$. graminicola genome was found to be repetitive. This indicates that the RepBase Update [16] fungal repeat dataset has very low coverage of fungalspecific repetitive sequences. RepBase Update [16] has limited sequence information with 256 sequences from 56 fungal species. This low repeat estimate may also reflect that most M. graminicola repetitive sequences are unique, as they were not found in other species. A similarity search of thirteen sequenced fungal genomes could only identify less than $1 \%$ of the repetitive sequences present in M. graminicola.

RM [15] was used in conjunction with repeat libraries derived from two repeat-finding approaches, RECON [17] and RepeatScout [18]. Of these two, the RECON [17] repeat library with a cutoff for high-copy repeats of 10 members/repeat family as used previously [17] was chosen for the subsequent in-depth analysis of the repetitive content of the genome to keep the number of families analyzed in detail reasonable. However, lowcopy repeat families (with 2-9 copies in the genome) also were annotated, of which only 125 families (0.05\%) could be assigned a useful annotation (Additional file 2: Table S2). The output from RECON [17] is primarily used for first-pass classification of repeats in newly sequenced genomes [17]. A comparison of the repetitive fraction identified by RECON [17] alone to RECON 


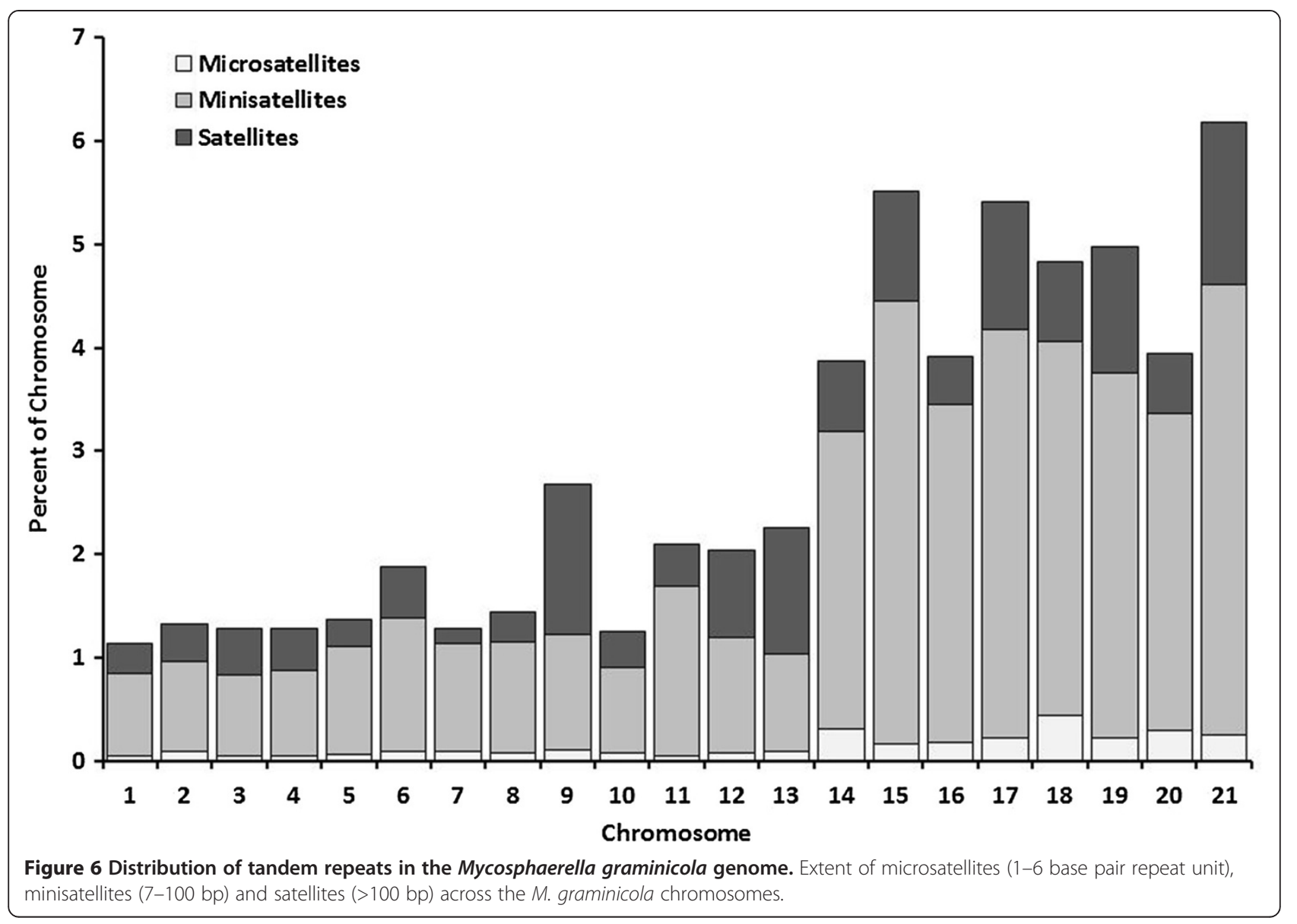

followed by RM [15], revealed that RM was able to identify similar but divergent elements that were missed by RECON (Table 1). RepeatScout [18] output reports a consensus sequence for each family, whereas RECON [17] extracts individual elements for each family, which not only makes the annotation step easier, but also accounts for the divergent members of the repeat families. Therefore, even though RepeatScout [18] predicted a slightly higher proportion (1.9\% more) of the M. graminicola genome as repetitive, we decided to annotate and report the RECON [17] output. The final repetitive fraction of $16.7 \%$ reported for $M$. graminicola is a conservative estimate as it excludes the low-complexity regions, simple repeats and repeat families containing fewer than 10 members/family. Adding all of those together estimated the total repetitive fraction at around $22.4 \%$ of the genome. The results from TALLYMER [20] were used for visualization purposes and correlated very well with the results from the other two methods (Figure 1). A decrease in percent GC content along the chromosome paralleled the occurrence of repetitive elements (Figure 1).

Relative insertion ages of 149 LTR retrotransposons that contained intact LTRs were estimated based on the occurrence of nucleotide substitutions between the 5' and 3' LTRs. However, the insertion timings may have been overestimated due to an elevated mutation rate as a consequence of RIP. Among the 149 retrotransposons with intact LTRs, only one element was found with identical sequences (insertion age of $0 \mathrm{My}$ ). Multiple sequence alignments of this element with other members of its family show mutated/RIPed sites in the internal region of this element but the LTRs themselves somehow escaped the effects of such mutations. This is interesting as this element would be an ideal candidate for future studies to investigate the timing and extent of RIP in subsequent cycles of sexual reproduction.

\section{Distribution of repetitive sequences}

Analysis of a single chromosome (chromosome 7) in Magnaporthe oryzae revealed a non-random distribution of repeats, with repetitive sequences occurring in three clusters mostly in heterochromatic regions near the telomeres [21]. This pattern however did not hold true in the completely sequenced $M$. graminicola genome, where the actual distribution of repetitive sequences across the whole genome and all of the chromosomes could be ascertained. Repetitive sequences in the M. graminicola 


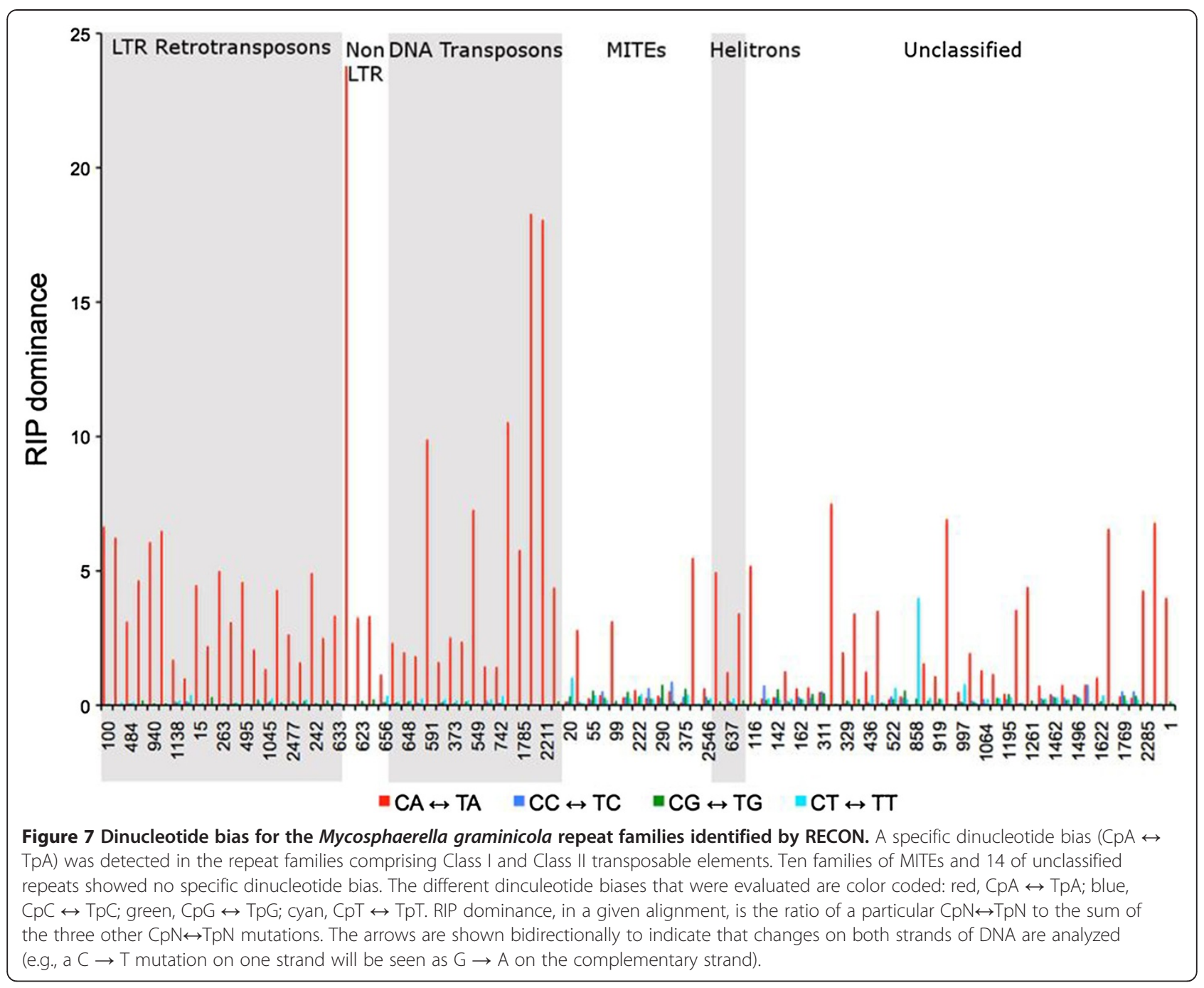

genome showed a non-random distribution across six chromosomes but were random for the remaining 15 .

Distribution of repetitive sequences and of transposable elements in particular can be random or clustered depending upon the insertion preferences of the different elements. For example, LINEs and SINEs in rodents and humans show an insertion preference to distinct chromosomal domains, leading to differences in their distribution patterns in their host genomes [22,23]. Our results show that both random and non-random distributions occur in the M. graminicola genome. The nested insertions primarily consisted of LTR-retrotransposons non-randomly clustered together in the gene-poor heterochromatic regions, as opposed to the smaller DNA transposons (MITEs) that were more prevalent in the gene-rich, euchromatic regions. Repeat-rich regions (AT-blocks), accounting for 36\% of the genome and 5\% of the gene space, are randomly distributed across the L. maculans supercontigs [24], whereas analyses of MITEs in the Epichloë genome revealed an insertion preference in the 5' regions of the genes [25]. A dissimilar distribution pattern of repetitive elements suggests that different TEs have evolved distinct strategies to persist in the genome.

The set of eight dispensable chromosomes was statistically significantly enriched in transposable elements and tandem repeats as compared to the core chromosomes. Dispensable chromosomes, also known as B or supernumerary chromosomes, were enriched for transposable elements in other fungi, including Fusarium oxysporum [26] and Nectaria haematococca [27]. However, unlike the M. graminicola genome, dispensable chromosomes in these other species were enriched for genes that played a major role in plant pathogenesis.

\section{Types of repetitive sequences}

Among all of the classes of repetitive elements, retrotransposons occupied the largest fraction in the M. graminicola genome. As retrotransposons follow a copy-paste mechanism for replication, these elements have usually been the 
major contributors to the repetitive fraction across a large number of genomes analyzed so far. The role of Class I retrotransposons in genome size inflation has been studied extensively in various organisms. In plants, a single family of LTR retrotransposon, BARE-1, is positively correlated to barley genome size increase [28], whereas genome size doubling of the wild rice Oryza australiensis was attributed to three LTR retrotransposon families that accounted for $60 \%$ of the genome [29]. Retrotransposons are also implicated in genome size expansion in fungi and oomycetes. The two Dothideomycetes relatives of $M$. graminicola with expanded genomes, Cladosporium fulvum [30] and $M$. fijiensis [31], had higher proportions of LTR retrotransposons in their genomes. The genome of the powdery mildew pathogen Blumeria graminis is four times larger than the average ascomycete genome; this difference can be attributed to non-LTR retrotransposons [32]. Similarly, in the oomycete $P$. infestans, two LTR retrotransposon families account for $29 \%$ of the $240-\mathrm{Mb}$ genome [9].

The M. gramincola retrotransposon families showed various stages of decay. There were six LTR retrotransposon families in which LTRs or TSDs could not be identified. The remaining LTR retrotransposon families also had truncated elements. Fragmented sequences that lack any retrotransposon structural features but show similarity to partial retrotransposon sequences have been termed 'remnants' [33]. Such remnants with small or large deletions have been observed in other organisms. Various mechanisms, such as illegitimate recombination in Arabidopsis [34], Saccharomyces cerevisiae [35] and Drosophila [36] and unequal homologous recombination in plants [33], are responsible for deletions in repetitive elements and tend to counteract the repetitive element expansion in the genome. The extent of remnants in a family is correlated to the age of the family [33], with older families having a larger number of truncated elements.

Three putative helitron families carrying helicase domains were identified but only two families had the hallmark structural features associated with helitrons. Occurrence of truncated elements in the third family made the identification of structural features impossible. Helitrons have been detected in other ascomycete fungi, such as Aspergillus nidulans [37] and Dothistroma pini [30]. An expansion of helitrons was observed in the $P$. infestans genome, which had 10-fold higher copies than the related P. ramorum and P. sojae genomes [9]. As compared to MITEs, TIRs and TSDs could not be identified for any of the DNA transposon families, which suggests that these transposon families may be old. Crypton, a class of DNA transposons found only in fungi [38] was also present in $M$. graminicola. These crypton sequences were initially identified by RECON [17] but were not included in the parsed set as they were below the family cut-off threshold of at least 10 elements.

\section{Telomeres, RecQ helicase and putative Penelope-like elements}

The RecQ helicase gene family, with extremely low GC content and tightly linked to the $M$. graminicola telomeres, has previously been identified in other fungal genomes including M. grisea [39], S. cerevisiae [40] and Ustilago maydis [41]. RecQ helicases are involved in a variety of functions including post-transcriptional gene silencing [42], maintaining genome stability [43] and DNA repair and recombination [44]. In S. cerevisiae, RecQ helicase is a part of the telomere-localized Y' element [45], which has been shown to play a structural role in protecting telomeres from accidental shortening or damage from recombination-mediated mechanisms. In S. cerevisiae strains lacking a gene for telomere replication, amplification and acquisition of $Y^{\prime}$ elements in a large number of telomeres decreases the frequency of cell death [46]. Besides a structural role, yeast RecQ helicase expression is induced during meiosis, suggesting a role in meiosis or sexual development [40].

Penelope-like elements (PLE), characterized by a bacterial GIY-YIG endonuclease domain, have a widespread distribution across the tree of life including other fungi, rotifers, plants and heterokonts [47]. One family of telomere-localized PLEs was identified in the M. graminicola genome. These putative PLEs are a subtype that localize to the subtelomeric regions in a characteristic orientation with telomeric repeats at their 3' ends [19]. Elements of this subtype lack the endonuclease domain but instead have species-specific telomeric repeats at their termini that can pair with the single-stranded telomere and utilize the 3' hydroxyl group for priming sequence synthesis [19]. Thus, these telomeric PLEs may play a role in protecting telomeres in addition to the regular telomerase enzyme, which in M. graminicola is located on chromosome 7. Telomeres in Drosophila [48], silkworm [49] and Giardia lamblia [50] are also maintained by non-LTR elements, but they carry an endonuclease domain. The telomeres in these three species consist of long arrays of non-LTR elements, unlike the endonuclease-lacking PLEs which are low-copy repeats.

\section{RIP in repetitive sequences}

Due to their inherent ability to amplify, defense mechanisms exist in the genome to minimize the numbers of transposons. One such mechanism that is specific to fungi is Repeat-Induced Point mutation (RIP). RIP targets multiple-copy sequences during meiosis and introduces cytosine $(C)$ to thymine $(T)$ mutations [51]. Sexual reproduction in the field is a common phenomenon in M. graminicola and ascospores have been shown to play a major role in long-distance disease spread and initiation [52]. Therefore, it seems highly likely that repetitive sequences in M. graminicola can be targeted by RIP 
during meiosis. Although RIP was not verified experimentally, a detailed analysis for all the repeat families revealed a higher number of transition mutations, supporting the existence of a RIP-like mechanism in $M$. graminicola, as noted previously [12,13]. However, our results were based on a much larger and global dataset as compared to the previous reports.

Certain dinucleotides are preferentially targeted by RIP, known as RIP bias, which varies with the organism [53]. Repetitive sequences in M. graminicola exhibited a CpA dinucleotide bias, the same as seen in Neurospora crassa [53]. However, ten families of MITEs and 14 families of unclassified elements did not show any clear dinucleotide bias. Repeat elements in these families were shorter than the minimum length threshold detected by the RIP machinery. RIP typically acts on sequences that are longer than $400 \mathrm{bp}$ in length [51] and are at least $80 \%$ identical [54]. Many families contained truncated elements and fewer full-length elements that might have prevented the detection of RIP bias in silico. Two completely identical $M$. graminicola rDNA repeat copies suggest that they were not detected by RIP. The presence of identical rDNA sequences is expected as these repeat clusters can evade RIP, although the exact mechanism is unknown [55].

The presence of transition mutations in the M. graminicola repetitive sequences generated many stop codons in the putative repeat-encoded protein domains. In $N$. crassa, RIP increased the occurrence of termination codons TAG and TAA [56]. However, a few elements in at least two families (families 623 and 18) were identified with no stop codons, although the presence of transition mutations in other parts of the elements indicated that the RIP machinery targeted these sequences. Lack of stop codons in the reverse-transcriptase domain suggested that these elements might still be active in the genome. Evidence for activity is also available at the transcript level, with at least two repetitive families (families 623 and 242) represented in the M. graminicola EST dataset. Therefore, these elements might be functional and active in the genome until they can be identified and targeted by the RIP mechanism during sexual reproduction.

\section{Nested clusters of transposable elements}

In a number of eukaryotic genomes, TEs are nested or inserted into other TEs $[57,58]$. Although many classes of TEs target specific regions in the genome, no obvious insertion preference for elements was identified in $M$. graminicola. As LTR retrotransposons were the most abundant in the M. graminicola genome, the majority of nested clusters identified had an LTR retrotransposon as the base element. One of the mechanisms by which LTR retrotransposons can target specific regions in the genome has been analyzed in Saccharomyces cerevisiae [59]. In S. cerevisiae, the Ty5 LTR retrotransposon targets telomeric heterochromatin as a result of direct interaction between retrotransposon-encoded integrase protein and the silent information regulator 4 protein (Sir4), associated with heterochromatin [59]. Nested clusters also have been reported in other fungi such as L. maculans [24] and Epichloë species [60], but were absent from other expanded fungal genomes, such as B. graminis [32]. Nested clusters also have been mentioned in $F$. oxysporum [61], N. crassa [62] and M. grisea [63-65], but they are mainly in the putative centromeric regions. The centromeres of M. graminicola have not been identified so whether they contain repeats is not known.

Due to their larger genome size and higher repetitive content, nesting of TEs has been studied extensively in plants. In maize, where $85 \%$ of the genome is repetitive [66] and ten retrotransposon families make up more than $25 \%$ of the genome, nested clusters may be a way to reduce the deleterious effects of TEs [57]. Genes were found in and near these nested TE clusters. Proliferation of a specific pathogenicity factor gene family unique to the powdery mildew pathogen $B$. graminis has been attributed to a family of LINE retrotransposons [67]. Such gene-repeat associations may hold an evolutionary and functional significance to the $M$. graminicola genome as it has been previously shown that in fungal and oomycete genomes, such repeat clusters act as 'breeding grounds', where new pathogenicity genes can be generated $[9,24,30]$.

\section{Conclusions}

Repetitive sequence analysis revealed that at least $16.7 \%$ of the M. graminicola genome was comprised of repeats. Dispensable chromosomes had a significantly higher repeat content as compared to core chromosomes. Class I elements occupied the largest fraction of the repetitive sequences in the M. graminicola genome. One family of telomere-localized Penelope-like elements was identified in the M. graminicola genome. The distribution of repeats was non-random on six chromosomes. Repeats in M. graminicola often were arranged in nested clusters of 2-7 elements. Even though all the transposable elements were riddled with transition mutations, there were putative transcriptionally-active elements in the $M$. graminicola genome as inferred from the absence of stop codons in EST sequences corresponding to at least three repeat families in the EST dataset.

\section{Methods}

\section{Identification of repeats}

Three methods were used to identify the repetitive sequences in M. graminicola. Repeats were identified de novo using RECON [17] and RepeatScout [18], a k-mer based method. The output from these two programs was 
used to generate custom repeat libraries, which were subsequently used to mask the $M$. graminicola genome using RepeatMasker [15]. Manually curated repeats specific to fungi were obtained from RepBase Update [16]. Repeats were also identified using another k-mer approach, TALLYMER [20], and frequencies of repetitive sequences were plotted along the chromosomes using Gnuplot [68].

\section{Annotation of repeats}

The repeat families identified by RECON [17] were annotated. The default output from RECON [17] was parsed to include families with 10 or more elements. The longest element from each family was compared against the NCBI non-redundant protein database using BLAST [69] to identify protein domains. These protein domains were used to classify repetitive sequences into different classes. Structural features such as Long Terminal Repeats (LTRs) and Terminal Inverted Repeats (TIRs) were verified in LTR retrotransposon and DNA transposon families using POLYDOT and EINVERTED, respectively, both from the EMBOSS [70] package. TIRs were also identified for families with no known protein sequences to identify Miniature Inverted Repeat Transposable Elements (MITEs). Sequences with no known proteins or other structural features were grouped into the unclassified category.

\section{Insertion age estimation for LTR retrotransposons}

Pair-wise alignment of intact LTRs from each LTR retrotransposon was used for estimating the age of insertion events. Numbers of substitutions were calculated for each pair and translated into divergence time using a substitution rate of $1.05 \times 10^{-9}$ nucleotides per site per year, as previously determined for fungi $[71,72]$.

\section{Distribution of repeats}

A non-parametric runs test for randomness was used to determine whether the repetitive sequences on the chromosomes occur in a pattern or are random. Each chromosome was divided into non-overlapping 100-kb bins and the extent of repetitive bases in each bin along with the average repetitive content/bin for the chromosome were calculated. The Lawstat package [73] in $\mathrm{R}$ [74] was used to do the analysis. The above steps were also repeated for non-overlapping, smaller $50-\mathrm{kb}$ bins. A similar analysis for gene distribution was done using 50-kb bins.

\section{Estimation of RIP}

Elements in each family were aligned using clustalX [75] and the alignments were manually curated using Jalview [76]. These alignments were used for estimating RIP dinucleotide bias using RIPcal [77]. For calculating percent identity, pairwise comparisons between elements of 15 repeat families were done. Elements with the highest GC content in each family were used as reference sequence. Sequence pairs with at least $99 \%$ sequence coverage were evaluated.

\section{Nested elements}

Relative chromosomal locations of the elements and their family annotations were used to identify clusters of nested elements. The base element was defined as the element into which all others were inserted. The alignment file for the base element family was used to determine if different parts of the base element made up a complete element. Target site duplication (TSD) of the base element in a given cluster was identified manually by examining the sequence in ARTEMIS [78].

\section{Subtelomere organization}

The distribution of different repetitive elements and proteins was analyzed over a 100-kb sequence from each end of the 21 chromosomes and viewed with OmniMapFree (http://www. omnimapfree.org). Repetitive family annotation was used to check for families exclusive to subtelomeric regions. For annotation of telomere-associated repeats and gene families, BLAST searches against the M. graminicola and NCBI 'nr' databases were done. The non-LTR retrotransposon reverse transcriptase domain was used to classify subtelomeric nonLTR retrotransposons using a web-based tool, RTclass1 [79].

\section{Tandem repeats}

Tandem Repeat Finder [80] was used to do whole-genome analyses to identify tandem repeats. Both the unmasked and masked (RM 'nolow' option) M. graminicola sequence was used for this analysis. The results were parsed using Tandem Repeat Analysis Program [81].

\section{Availability of supporting data}

The data sets supporting the results of this article are available in the LabArchives, LLC, repository, DOI 10.6070/H4222RRG [unique persistent identifier and hyperlink to dataset(s) in http:// format will be submitted and provided if accepted].

\section{Endnote}

Names are necessary to report factually on available data. However, the USDA neither guarantees nor warrants the standard of the product, and the use of the name implies no approval of the product to the exclusion of others that also may be suitable.

\section{Additional files}

Additional file 1: Table S1. Repetitive families identified in Mycosphaerella graminicola by RECON. A summary of the general features of repetitive families identified in the finished genome sequence of $M$. graminicola by 
RECON. These features include family number, family name, element copy number, base pair coverage, annotation, families that were merged together, LTR/R length, TSD length, presence/absence of RIP and RIP index value.

Additional file 2: Table S2. Repetitive families with fewer than 10 repeat elements per family identified in the Mycosphaerella graminicola genome. Repeat classification for 125 repetitive families, which had fewer than 10 elements per family in the M. graminicola genome.

Additional file 3: Table S3. Runs test for randomness of repetitive sequences across the Mycosphaerella graminicola chromosomes. This table lists the consecutive runs of $0 \mathrm{~s}$ (repetitive content in a nonoverlapping $50-k b$ bin less than the chromosomal average) and $1 \mathrm{~s}$ (repetitive content of the 50-kb bin is above the chromosomal average).

\section{Competing interests}

The authors declare that they have no competing interests.

\section{Authors' contributions}

$\mathrm{BD}$ and $\mathrm{SBG}$ designed the analysis. BD performed the analysis and wrote the initial draft. $\mathrm{BD}, \mathrm{NG}, \mathrm{RH}$ and $\mathrm{SBG}$ edited and finalized the manuscript. All authors read and approved the final manuscript.

\section{Acknowledgements}

DNA sequencing of $M$. graminicola was performed at the U. S. Department of Energy's Joint Genome Institute through the Community Sequencing Program (www.jgi.doe.gov/csp/) and all sequence data are publicly available. Supported by USDA CRIS project 3602-22000-015-00D. BD and RCH were supported by Genome Canada Large Scale Applied Research Project 164DIA.

\section{Author details}

'Department of Forest and Conservation Sciences, 2424 Main Mall, Vancouver, BC V6T 1Z4, Canada. ${ }^{2}$ Department of Botany, Beaty Biodiversity Centre, 2212 Main Mall, Vancouver, BC V6T 1Z4, Canada. ${ }^{3}$ Natural Resources Canada, Laurentian Forestry Centre, 1055 du PEPS, Stn. Sainte-Foy, P.O. Box 10380, Quebec, QC G1V 4C7, Canada. ${ }^{4}$ USDA-ARS, Crop Production and Pest Control Research Unit, Purdue University, 915 W. State Street, West Lafayette, Indiana 47907-2054, USA.

Received: 1 August 2014 Accepted: 12 December 2014 Published: 17 December 2014

\section{References}

1. Palmer C-L, Skinner W: Mycosphaerella graminicola: latent infection, crop devastation and genomics. Mol Plant Pathol 2002, 3(2):63-70.

2. Goodwin S: Back to basics and beyond: increasing the level of resistance to Septoria tritici blotch in wheat. Australas Plant Pathol 2007, 36(6):532-538.

3. Fisher N, Griffin M: Benzimidazole (MBC) resistance in Septoria tritici. ISPP Chem Control News/ 1984, 5:8-9.

4. Fraaije BA, Burnett FJ, Clark WS, Motteram J, Lucas JA: Resistance development to Qol inhibitors in populations of Mycosphaerella graminicola in the UK. In Modern fungicides and antifungal compounds $N$. Edited by Dehne HW, Gisi U, Kuck KH, Russell PE, Alton LH. UK: British Crop Protection Council; 2005:63-71.

5. Mycosphaerella graminicola genome portal. http://genome.jgi-psf.org/ Mycgr3/Mycgr3.home.html.

6. Wicker T, Sabot F, Hua-Van A, Bennetzen JL, Capy P, Chalhoub B, Flavell A, Leroy P, Morgante M, Panaud O, Paux E, SanMiguel P, Schulman AH: A unified classification system for eukaryotic transposable elements. Nat Rev Genet 2007, 8(12):973-982.

7. McClintock B: The origin and behavior of mutable loci in maize. Proc Natl Acad Sci 1950, 36(6):344-355.

8. Kazazian HH: Mobile elements: drivers of genome evolution. Science 2004, 303(5664):1626-1632.

9. Haas BJ, Kamoun S, Zody MC, Jiang RHY, Handsaker RE, Cano LM, Grabherr M, Kodira CD, Raffaele S, Torto-Alalibo T, Bozkurt TO, Ah-Fong AMV, Alvarado L, Anderson VL, Armstrong MR, Avrova A, Baxter L, Beynon J, Boevink PC, Bollmann SR, Bos JIB, Bulone V, Cai G, Cakir C, Carrington JC, Chawner M, Conti $L$, Costanzo S, Ewan R, Fahlgren N et al: Genome sequence and analysis of the Irish potato famine pathogen Phytophthora infestans. Nature 2009, 461(7262):393-398.
10. Jiang N, Bao Z, Zhang X, Eddy SR, Wessler SR: Pack-MULE transposable elements mediate gene evolution in plants. Nature 2004, 431(7008):569-573.

11. Xiao $H$, Jiang $N$, Schaffner $E$, Stockinger EJ, van der Knaap E: A retrotransposon-mediated gene duplication underlies morphological variation of tomato fruit. Science 2008, 319(5869):1527-1530.

12. Dhillon B, Cavaletto JR, Wood KV, Goodwin SB: Accidental amplification and inactivation of a methyltransferase gene eliminates cytosine methylation in Mycosphaerella graminicola. Genetics 2010, 186(1):67-77.

13. Goodwin SB, Ben M'Barek S, Dhillon B, Wittenberg AHJ, Crane CF, Hane JK, Foster AJ, Van der Lee TAJ, Grimwood J, Aerts A, Antoniw J, Bailey A, Bluhm B, Bowler J, Bristow J, van der Burgt A, Canto-Canché B, Churchill ACL, Conde-Ferràez L, Cools HJ, Coutinho PM, Csukai M, Dehal P, De Wit P, Donzelli B, van de Geest HC, van Ham RCHJ, Hammond-Kosack KE, Henrissat B, Kilian A et al: Finished genome of the fungal wheat pathogen Mycosphaerella graminicola reveals dispensome structure, chromosome plasticity, and stealth pathogenesis. PLOS Genet 2011, 7(6):e1002070.

14. Wittenberg AHJ, van der Lee TAJ, Ben M'Barek S, Ware SB, Goodwin SB, Kilian A, Visser RGF, Kema GHJ, Schouten HJ: Meiosis drives extraordinary genome plasticity in the haploid fungal plant pathogen Mycosphaerella graminicola. PLOS ONE 2009, 4(6):e5863.

15. Smit AFA, Hubley R, Green P: RepeatMasker Open3.0. 1996-2004 [http:// repeatmasker.org]

16. Jurka J: Repbase update: a database and an electronic journal of repetitive elements. Trends Genet 2000, 16(9):418-420.

17. Bao Z, Eddy SR: Automated de novo identification of repeat sequence families in sequenced genomes. Genome Res 2002, 12(8):1269-1276.

18. Price $A L$, Jones NC, Pevzner PA: De novo identification of repeat families in large genomes. Bioinformatics 2005, 21(Suppl 1):i351-i358.

19. Gladyshev EA, Arkhipova IR: Telomere-associated endonuclease-deficient Penelope-like retroelements in diverse eukaryotes. Proc Natl Acad Sci 2007, 104(22):9352-9357

20. Kurtz S, Narechania A, Stein JC, Ware D: A new method to compute K-mer frequencies and its application to annotate large repetitive plant genomes. BMC Genomics 2008, 9:517.

21. Thon M, Pan H, Diener S, Papalas J, Taro A, Mitchell T, Dean R: The role of transposable element clusters in genome evolution and loss of synteny in the rice blast fungus Magnaporthe oryzae. Genome Biol 2006, 7(2):R16.

22. Chen T, Manuelidis L: SINEs and LINEs cluster in distinct DNA fragments of Giemsa band size. Chromosoma 1989, 98(5):309-316.

23. Acosta MJ, Marchal JA, Fernandez-Espartero CH, Bullejos M, Sanchez A: Retroelements (LINEs and SINEs) in vole genomes: Differential distribution in the constitutive heterochromatin. Chromosom Res 2008, 16(7):949-959.

24. Rouxel T, Grandaubert J, Hane JK, Hoede C, van de Wouw AP, Couloux A, Dominguez V, Anthouard V, Bally P, Bourras S, Cozijnsen AJ, Ciuffetti LM, Degrave A, Dilmaghani A, Duret L, Fudal I, Goodwin SB, Gout L, Glaser N, Linglin J, Kema GHJ, Lapalu N, Lawrence CB, May K, Meyer M, Ollivier B, Poulain J, Schoch CL, Simon A, Spatafora JW, et al: Effector diversification within compartments of the Leptosphaeria maculans genome affected by Repeat-Induced Point mutations. Nat Commun 2011, 2:202.

25. Fleetwood DJ, Khan AK, Johnson RD, Young CA, Mittal S, Wrenn RE, Hesse U, Foster SJ, Schardl CL, Scott B: Abundant degenerate miniature inverted-repeat transposable elements in genomes of Epichloid fungal endophytes of grasses. Genome Biol Evol 2011, 3:1253-1264.

26. Ma L-J, van der Does HC, Borkovich KA, Coleman JJ, Daboussi M-J, Di Pietro A, Dufresne M, Freitag M, Grabherr M, Henrissat B, Houterman PM, Kang S, Shim W-B, Woloshuk C, Xie X, Xu J-R, Antoniw J, Baker SE, Bluhm BH, Breakspear A, Brown DW, Butchko RAE, Chapman S, Coulson R, Coutinho PM, Danchin EGJ Diener A, Gale LR, Gardiner DM, Goff S, et al: Comparative genomics reveals mobile pathogenicity chromosomes in Fusarium. Nature 2010, 464(7287):367-373.

27. Coleman JJ, Rounsley SD, Rodriguez-Carres M, Kuo A, Wasmann CC, Grimwood J, Schmutz J, Taga M, White GJ, Zhou S, Schwartz DC, Freitag M, Ma L-J, Danchin EGJ, Henrissat B, Coutinho PM, Nelson DR, Straney D, Napoli CA, Barker BM, Gribskov M, Rep M, Kroken S, Molnár I, Rensing C, Kennell JC, Zamora J, Farman ML, Selker EU, Salamov A, et al: The Genome of Nectria haematococca: Contribution of Supernumerary Chromosomes to Gene Expansion. PLoS Genet 2009, 5(8):e1000618.

28. Kalendar R, Tanskanen J, Immonen S, Nevo E, Schulman AH: Genome evolution of wild barley (Hordeum spontaneum) by BARE-1 retrotransposon dynamics in response to sharp microclimatic divergence. Proc Natl Acad Sci U S A 2000, 97(12):6603-6607. 
29. Piegu B, Guyot R, Picault N, Roulin A, Saniyal A, Kim H, Collura K, Brar DS, Jackson S, Wing RA, Panaud O: Doubling genome size without polyploidization: dynamics of retrotransposition-driven genomic expansions in Oryza australiensis, a wild relative of rice. Genome Res 2006, 16(10):1262-1269.

30. de Wit PJGM, van der Burgt A, Okmen B, Stergiopoulos I, Abd-Elsalam KA, Aerts AL, Bahkali AH, Beenen HG, Chettri P, Cox MP, Datema E, de Vries RP, Dhillon B, Ganley AR, Griffiths SA, Guo Y, Hamelin RC, Henrissat B, Kabir MS, Jashni MK, Kema G, Klaubauf S, Lapidus A, Levasseur A, Lindquist E, Mehrabi R, Ohm RA, Owen TJ, Salamov A, Schwelm A, et al: The genomes of the fungal plant pathogens Cladosporium fulvum and Dothistroma septosporum reveal adaptation to different hosts and lifestyles but also signatures of common ancestry. PLoS Genet 2012, 8(11):e1003088.

31. Ohm RA, Feau N, Henrissat B, Schoch CL, Horwitz BA, Barry KW, Condon BJ, Copeland AC, Dhillon B, Glaser F, Hesse CN, Kosti I, LaButti K, Lindquist EA, Lucas S, Salamov AA, Bradshaw RE, Ciuffetti L, Hamelin RC, Kema GHJ, Lawrence C, Scott JA, Spatafora JW, Turgeon BG, de Wit PJGM, Zhong S, Goodwin SB, Grigoriev IV: Diverse lifestyles and strategies of plant pathogenesis encoded in the genomes of eighteen Dothideomycetes fungi. PLOS Pathog 2012, 8(12):e1003037.

32. Spanu PD, Abbott JC, Amselem J, Burgis TA, Soanes DM, Stuber K, van Themaat EV L, Brown JKM, Butcher SA, Gurr SJ, Lebrun M-H, Ridout CJ, Schulze-Lefert P, Talbot NJ, Ahmadinejad N, Ametz C, Barton GR, Benjdia M, Bidzinski P, Bindschedler LV, Both M, Brewer MT, Cadle-Davidson L, CadleDavidson MM, Collemare J, Cramer R, Frenkel O, Godfrey D, Harriman J, Hoede $C$, et al: Genome expansion and gene loss in powdery mildew fungi reveal tradeoffs in extreme parasitism. Science 2010, 330(6010):1543-1546.

33. Ma J, Devos KM, Bennetzen JL: Analyses of LTR-retrotransposon structures reveal recent and rapid genomic DNA loss in rice. Genome Res 2004 14(5):860-869.

34. Devos KM, Brown JK, Bennetzen JL: Genome size reduction through illegitimate recombination counteracts genome expansion in Arabidopsis. Genome Res 2002, 12(7):1075-1079.

35. Asami Y, Jia DW, Tatebayashi K, Yamagata K, Tanokura M, Ikeda H: Effect of the DNA topoisomerase II inhibitor VP-16 on illegitimate recombination in yeast chromosomes. Gene 2002, 291(1-2):251-257.

36. Petrov DA, Lozovskaya ER, Hartl DL: High intrinsic rate of DNA loss in Drosophila. Nature 1996, 384(6607):346-349.

37. Galagan JE, Calvo SE, Cuomo C, Ma L-J, Wortman JR, Batzoglou S, Lee S-I, Basturkmen M, Spevak CC, Clutterbuck J, Kapitonov V, Jurka J, Scazzocchio C, Farman M, Butler J, Purcell S, Harris S, Braus GH, Draht O, Busch S, D'Enfert C, Bouchier C, Goldman GH, Bell-Pedersen D, Griffiths-Jones S, Doonan JH, Yu J, Vienken K, Pain A, Freitag M, et al: Sequencing of Aspergillus nidulans and comparative analysis with A. fumigatus and A. oryzae. Nature 2005, 438(7071):1105-1115.

38. Goodwin TJ, Butler MI, Poulter RT: Cryptons: a group of tyrosine-recombinaseencoding DNA transposons from pathogenic fungi. Microbiology 2003, 149(Pt 11):3099-3109.

39. Gao W, Khang CH, Park SY, Lee YH, Kang S: Evolution and organization of a highly dynamic, subtelomeric helicase gene family in the rice blast fungus Magnaporthe grisea. Genetics 2002, 162(1):103-112.

40. Louis EJ: The chromosome ends of Saccharomyces cerevisiae. Yeast 1995, 11(16):1553-1573.

41. Sanchez-Alonso P, Guzman P: Organization of chromosome ends in Ustilago maydis. RecQ-like helicase motifs at telomeric regions. Genetics 1998, 148(3):1043-1054.

42. Cogoni C, Macino G: Posttranscriptional gene silencing in Neurospora by a RecQ DNA helicase. Science 1999, 286(5448):2342-2344.

43. Chakraverty RK, Hickson ID: Defending genome integrity during DNA replication: a proposed role for RecQ family helicases. Bioessays 1999, 21(4):286-294

44. Shen JC, Loeb LA: The Werner syndrome gene: the molecular basis of RecQ helicase-deficiency diseases. Trends Genet 2000, 16(5):213-220.

45. Louis EJ, Naumova ES, Lee A, Naumov G, Haber JE: The chromosome end in yeast: its mosaic nature and influence on recombinational dynamics. Genetics 1994, 136(3):789-802.

46. Lundblad V, Blackburn EH: An alternative pathway for yeast telomere maintenance rescues est1- senescence. Cell 1993, 73(2):347-360.

47. Arkhipova IR: Distribution and phylogeny of Penelope-like elements in eukaryotes. Syst Biol 2006, 55(6):875-885.
48. George JA, DeBaryshe PG, Traverse KL, Celniker SE, Pardue M-L: Genomic organization of the Drosophila telomere retrotransposable elements. Genome Res 2006, 16(10):1231-1240.

49. Fujiwara H, Osanai M, Matsumoto T, Kojima K: Telomere-specific non-LTR retrotransposons and telomere maintenance in the silkworm, Bombyx mori. Chromosome Res 2005, 13(5):455-467.

50. Arkhipova IR, Morrison HG: Three retrotransposon families in the genome of Giardia lamblia: Two telomeric, one dead. Proc Natl Acad Sci 2001, 98(25):14497-14502.

51. Watters MK, Randall TA, Margolin BS, Selker EU, Stadler DR: Action of repeat-induced point mutation on both strands of a duplex and on tandem duplications of various sizes in Neurospora. Genetics 1999, 153(2):705-714.

52. Garcia C, Marshall D: Observations on the ascogenous stage of Septoria tritici in Texas. Mycol Res 1992, 96:65-70.

53. Cambareri EB, Jensen BC, Schabtach E, Selker EU: Repeat-induced G-C to A-T mutations in Neurospora. Science 1989, 244(4912):1571-1575.

54. Cambareri EB, Singer MJ, Selker EU: Recurrence of repeat-induced point mutation (RIP) in Neurospora crassa. Genetics 1991, 127(4):699-710.

55. Galagan JE, Selker EU: RIP: the evolutionary cost of genome defense. Trends Genet 2004, 20(9):417-423.

56. Singer MJ, Marcotte BA, Selker EU: DNA methylation associated with repeat-induced point mutation in Neurospora crassa. Mol Cell Biol 1995. 15(10):5586-5597.

57. SanMiguel P, Tikhonov A, Jin YK, Motchoulskaia N, Zakharov D, Melake-Berhan A, Springer PS, Edwards KJ, Lee M, Avramova Z, Bennetzen JL: Nested retrotransposons in the intergenic regions of the maize genome. Science 1996, 274(5288):765-768.

58. Baucom RS, Estill JC, Chaparro C, Upshaw N, Jogi A, Deragon J-M, Westerman RP, SanMiguel PJ, Bennetzen JL: Exceptional diversity, non-random distribution, and rapid evolution of retroelements in the B73 maize genome. PLOS Genet 2009, 5(11):e1000732.

59. Xie W, Gai X, Zhu Y, Zappulla DC, Sternglanz R, Voytas DF: Targeting of the yeast Ty5 retrotransposon to silent chromatin is mediated by interactions between integrase and Sir4p. Mol Cell Biol 2001, 21(19):6606-6614.

60. Schardl CL, Young CA, Hesse U, Amyotte SG, Andreeva K, Calie PJ, Fleetwood DJ, Haws DC, Moore N, Oeser B, Panaccione DG, Schweri KK, Voisey CR, Farman ML, Jaromczyk JW, Roe BA, O'Sullivan DM, Scott B, Tudzynski P, An Z, Arnaoudova EG, Bullock CT, Charlton ND, Chen L, Cox M, Dinkins RD, Florea S, Glenn AE, Gordon A, Güldener U, et al: Plant-symbiotic fungi as chemical engineers: multi-genome analysis of the Clavicipitaceae reveals dynamics of alkaloid loci. PLoS Genet 2013, 9(2):e1003323.

61. Hua-Van A, Daviere JM, Kaper F, Langin T, Daboussi MJ: Genome organization in Fusarium oxysporum: clusters of class II transposons. Curr Genet 2000, 37(5):339-347.

62. Cambareri EB, Aisner R, Carbon J: Structure of the chromosome VII centromere region in Neurospora crassa: degenerate transposons and simple repeats. Mol Cell Biol 1998, 18(9):5465-5477.

63. Valent B, Chumley FG: Avirulence genes and mechanisms of genetic instability in the rice blast fungus. In The Rice Blast Disease. Edited by Zeigler RS, Leong SA, Teng PS. Cambridge, UK: Oxford University Press; 1994:111-134.

64. Nitta N, Farman ML, Leong SA: Genome organization of Magnaporthe grisea: integration of genetic maps, clustering of transposable elements and identification of genome duplications and rearrangements. TAG Theor Appl Genet 1997, 95(1):20-32.

65. Dean RA, Talbot NJ, Ebbole DJ, Farman ML, Mitchell TK, Orbach MJ, Thon M, Kulkarni R, Xu JR, Pan H, Read ND, Lee YH, Carbone I, Brown D, Oh YY, Donofrio N, Jeong JS, Soanes DM, Djonovic S, Kolomiets E, Rehmeyer C, Li W, Harding M, Kim S, Lebrun MH, Bohnert H, Coughlan S, Butler J, Calvo S, Ma Let al: The genome sequence of the rice blast fungus Magnaporthe grisea. Nature 2005, 434(7036):980-986.

66. Schnable PS, Ware D, Fulton RS, Stein JC, Wei F, Pasternak S, Liang C, Zhang J, Fulton L, Graves TA, Minx P, Reily AD, Courtney L, Kruchowski SS, Tomlinson C, Strong C, Delehaunty K, Fronick C, Courtney B, Rock SM, Belter E, Du F, Kim K, Abbott RM, Cotton M, Levy A, Marchetto P, Ochoa K, Jackson SM, Gillam B, et al: The $B 73$ maize genome: complexity, diversity, and dynamics. Science 2009, 326(5956):1112-1115.

67. Sacristan S, Vigouroux M, Pedersen C, Skamnioti P, Thordal-Christensen H, Micali C, Brown JKM, Ridout CJ: Coevolution between a family of parasite virulence effectors and a class of LINE-1 retrotransposons. PLOS ONE 2009, 4(10):e7463. 
68. Williams T, Kelley C: Gnuplot 4.4: an interactive plotting program. 2010 [http://gnuplot.sourceforge.net/]

69. Altschul SF, Gish W, Miller W, Myers EW, Lipman DJ: Basic local alignment search tool. J Mol Biol 1990, 215(3):403-410.

70. Rice P, Longden I, Bleasby A: EMBOSS: the European Molecular Biology Open Software Suite. Trends Genet 2000, 16(6):276-277.

71. Berbee ML, Taylor JW: Dating the molecular clock in fungi - how close are we? Fungal Biol Rev 2010, 24(1-2):1-16.

72. Kasuga T, White TJ, Taylor JW: Estimation of nucleotide substitution rates in Eurotiomycete fungi. Mol Biol Evol 2002, 19(12):2318-2324.

73. Noguchi K, Hui WLW, Gel YR, Gastwirth JL, Miao W: lawstat: An R package for biostatistics, public policy, and law. 2009 [http://CRAN.R-project.org/ package=lawstat]

74. R Development Core Team: R: A language and environment for statistical computing. 2011 [http://www.R-project.org]

75. Larkin MA, Blackshields G, Brown NP, Chenna R, McGettigan PA, McWilliam $H$, Valentin F, Wallace IM, Wilm A, Lopez R, Thompson JD, Gibson TJ, Higgins DG: Clustal W and Clustal X version 2.0. Bioinformatics 2007, 23(21):2947-2948

76. Clamp M, Cuff J, Searle SM, Barton GJ: The Jalview Java alignment editor. Bioinformatics 2004, 20(3):426-427.

77. Hane JK, Oliver RP: RIPCAL: a tool for alignment-based analysis of repeatinduced point mutations in fungal genomic sequences. BMC Bioinformatics 2008, 9:478.

78. Rutherford K, Parkhill J, Crook J, Horsnell T, Rice P, Rajandream MA, Barrell B: Artemis: sequence visualization and annotation. Bioinformatics 2000, 16(10):944-945.

79. Kapitonov W, Tempel S, Jurka J: Simple and fast classification of non-LTR retrotransposons based on phylogeny of their RT domain protein sequences. Gene 2009, 448(2):207-213.

80. Benson G: Tandem repeats finder: a program to analyze DNA sequences. Nucleic Acids Res 1999, 27(2):573-580.

81. Sobreira TJ, Durham AM, Gruber A: TRAP: automated classification, quantification and annotation of tandemly repeated sequences. Bioinformatics 2006, 22(3):361-362.

doi:10.1186/1471-2164-15-1132

Cite this article as: Dhillon et al.: The landscape of transposable elements in the finished genome of the fungal wheat pathogen Mycosphaerella graminicola. BMC Genomics 2014 15:1132.

\section{Submit your next manuscript to BioMed Central and take full advantage of:}

- Convenient online submission

- Thorough peer review

- No space constraints or color figure charges

- Immediate publication on acceptance

- Inclusion in PubMed, CAS, Scopus and Google Scholar

- Research which is freely available for redistribution

Submit your manuscript at www.biomedcentral.com/submit 